\title{
Advective Displacement Method for the Characterisation of Pore Water Chemistry and Transport Properties in Claystone
}

\author{
Urs Mäder (iD \\ Geological Sciences, University of Bern, Baltzerstrasse 3, 3067 Bern, Switzerland \\ Correspondence should be addressed to Urs Mäder; urs.maeder@geo.unibe.ch \\ Received 6 July 2017; Accepted 4 December 2017; Published 14 January 2018 \\ Academic Editor: Jean-Michel Matray
}

Copyright ( 2018 Urs Mäder. This is an open access article distributed under the Creative Commons Attribution License, which permits unrestricted use, distribution, and reproduction in any medium, provided the original work is properly cited.

\begin{abstract}
The advective displacement method applies large hydraulic gradients to a confined rock core sample to yield small aliquots of the preserved in situ pore water, applicable to aquitard rocks with hydraulic conductivities as low as $10^{-14} \mathrm{~m} / \mathrm{s}$. Examples from argillaceous rocks indicate that only minor artefacts are induced and that analytical methods optimized for small aliquots provide a comprehensive chemical and isotopic characterisation. Multicomponent transport properties are derived from extending experimental time and using a traced artificial pore water for injection. Examples include quantification of the anion exclusion effect that can even resolve a small difference between transport properties for chloride and bromide in claystone. Controls by mineral saturation and cation exchange processes are also constrained by data from this approach. Technical details are provided for construction, material selection, components, sensors, and analytical issues.
\end{abstract}

\section{Introduction}

Properties and performance of hydraulically tight clayey rock formations are of interest in the context of cap rocks for oil, gas, and carbon dioxide confinement, as pore water archives for past hydrogeochemical evolution, as hydrocarbon source rock for shale gas, and as confining units for radioactive and chemotoxic waste ([1, 2], editorial of this volume). In contrast to tight crystalline rocks, porosity and water content are substantial, in the range of a few to about twenty volume percent, and hydraulic conductivities are typically $<10^{-12} \mathrm{~m} / \mathrm{s}$. The focus of this paper is on a particular method from which to gain insight into pore water chemistry, hydraulic properties, transport of dissolved species, and reactive transport (water/rock interaction) that may also be coupled to the hydraulic properties. The motivation is to gain process understanding in the context of deep geologic disposal of radioactive waste, but the methods and results are transferable to the other aforementioned fields of research.

Our understanding of pore water chemistry and transport of dissolved species is based on models that bridge measureable quantities (fluxes, concentrations, and bulk properties) and the nanometric pore scale that is only very selectively observable. A milestone was the Pearson et al. [3] data digest on Opalinus Clay based on laboratory work and field tests performed in the Underground Rock Laboratory at Mont Terri, Switzerland (https://www.mont-terri.ch). The thermodynamically based concepts of aqueous speciation, mineral equilibria (and buffering), interdependencies such as $\mathrm{pH}-\mathrm{P}_{\mathrm{CO}_{2}}$-carbonate-sulphate-ion exchanger [4], and electrostatic effects exerted by certain clay minerals (commonly named "anion exclusion" based on some variant of Poisson-Boltzmann theory) offer a sufficient level of complexity for the discussion of results presented in this paper. Significant progress has been made regarding details, and more constraining data have been elaborated [5-8].

Sophisticated reactive transport models (e.g., Crunchflow, Flotran, and Phreeqc-Comsol coupling) and inexpensive computation power are available to simulate fully described laboratory experiments that are necessary as testing cases for both implementation of complex and coupled processes (benchmark-type exercises) and testing of alternative conceptualisations (choice of processes and simplifying boundary/initial conditions). An example of such a data set and its comparative modelling by increasing degrees of complexity is that of a reactive transport core infiltration experiment in compacted bentonite [9]. 
The focus of this contribution is on the technical details of the experimental approach because much hinges on design details and material selection for equipment, on details of sample preparation, on the ability to analyse very small samples, and on keeping experiments stable for months to years.

\section{Materials and Methods}

2.1. Advective Displacement Method and Sample Requirement. During the 1990s when characterisation of pore water in tight clayey rock formations was advancing, direct sampling techniques included rock squeezing, long-term borehole sampling, and an indirect fingerprinting method by aqueous leaching [3]. As an alternative, a so-called advective displacement method was tested whereby the injection of an artificial pore water on one side of a sample core was used to displace the in situ pore water out of the other end of the sample [10]. A first pore water displacement experiment with Opalinus Clay started in 2002, and it is still running as of 2017. The original apparatus was constructed at the Paul Scherrer Institut (Laboratory for Waste Management), Switzerland, for the purpose of studying radionuclide transport in synthetic fractures, and this prototype was moved to the University of Bern and subsequently modified and replaced by new equipment designed and constructed in-house.

The advective displacement method implicitly assumes the following:

(i) A cylindrical rock sample with planar ends perpendicular to the cylinder axis

(ii) A well-preserved rock sample, with respect to water content and the reactive mineral components (pyrite), for example, sealed in moderate vacuum and stored refrigerated or at in situ temperature of the formation

(iii) No disturbing effects from drilling fluid (particularly if stable isotope data should be acquired), or other cutting fluids, or from compressed gas when dry drilling is employed (for some argillaceous rocks). The outermost near-surface part may be turned off efficiently on a lathe

(iv) Minimization of exposure to atmosphere at all stages

(v) Approximation of $1 \mathrm{D}$ column transport. This is normally met by samples taken perpendicular to bedding but may not be met for samples parallel to bedding and that show distinct layering. In the latter case, a more permeable subvolume may be tested preferentially.

A number of potentially disturbing effects are difficult to control:

(i) Volumetric strain is associated with the unloading of a sample when sampling a deep formation.

(ii) Outgassing will occur in case gas saturation is reached during unloading.

(iii) The presence of a gas phase at in situ condition will result in gas escape and potentially some displacement of pore water. (iv) An adjustment of pore water chemistry from in situ temperature to storage or experimental temperature will occur (carbonate-sulphate system).

(v) Microbial activity may start at sample surfaces and may persist during an experiment.

2.2. Properties and Processes That Can Be Studied by Advective Displacement Experiments. Diverse processes, chemical, physical, and transport properties, can be determined by imposing a wide range of hydraulic and chemical boundary conditions for advective displacement experiments.

(1) Hydraulic conductivity $K[\mathrm{~m} / \mathrm{s}]$ is calculated very accurately from sample dimensions $\left(l[\mathrm{~m}], A\left[\mathrm{~m}^{2}\right]\right)$, average hydraulic head $(h[\mathrm{~m}])$, and average volumetric flow rate $\left(Q\left[\mathrm{~m}^{3} / \mathrm{s}\right]\right)$ for a time span of interest, $K=(Q \cdot l) /(A \cdot h)$. A lower practical limit is $10^{-14} \mathrm{~m} / \mathrm{s}$ if fluid chemistry is also of interest, or even $10^{-15} \mathrm{~m} / \mathrm{s}$ if only conductivity is to be determined.

(2) The temperature dependence of hydraulic conductivity can be determined accurately with a thermostatequipped device.

(3) Changes of hydraulic conductivity as function of total stress of a sample or clogging of flow-paths by transport of micro particles or by precipitation/dissolution of secondary minerals (fluid-rock interaction) can also be recorded.

(4) The in situ pore water can be displaced with an artificial pore water, and the first few small samples represent pore water composition, subject to some artefacts.

(5) Extending pore water displacement to an advection experiment with tracers, breakthrough of these can be analysed and transport properties determined, such as anions versus deuterated water, or reactive components such as cations, or dissolved organic carbon species.

(6) In very reactive systems (soluble salts), changes in porosity and/or pore structure will affect hydraulic properties, and this can be monitored.

(7) Anion-accessible porosity ratio can be determined as the ratio between the anion concentration obtained from an aqueous extract back-calculated to the total porosity (water content) of a sample and that measured in the outflow. This assumes that the anionaccessible porosity is represented by the porosity that is also involved in advective flow.

(8) Because outflow can be continuously sampled, the saturation state with respect to potentially controlling minerals (precipitation or dissolution) can be evaluated as a function of percolation time. These are clues for potentially controlling mineral equilibria (carbonates, sulphates, but also ion-exchange).

(9) Postmortem analysis of a sample core may yield additional constraints apart from physical properties, such as a longitudinal distribution of the cation 


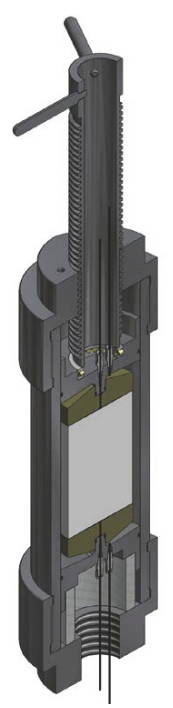

(a)

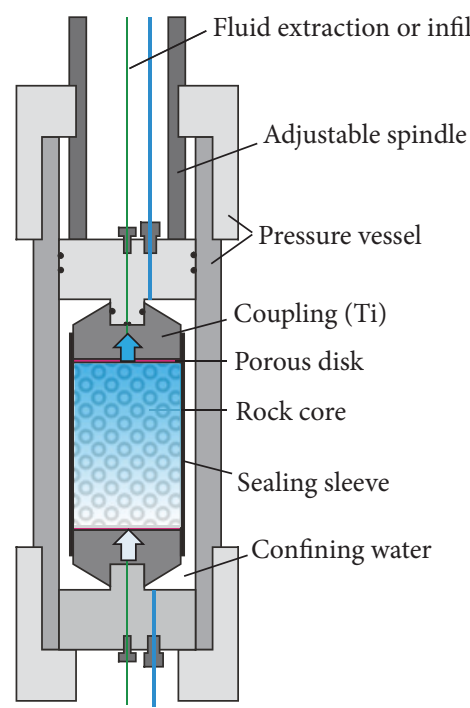

(b)

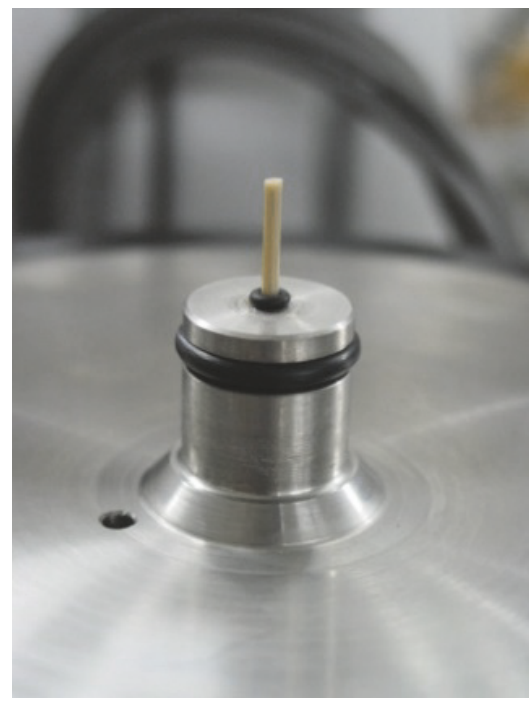

(d)

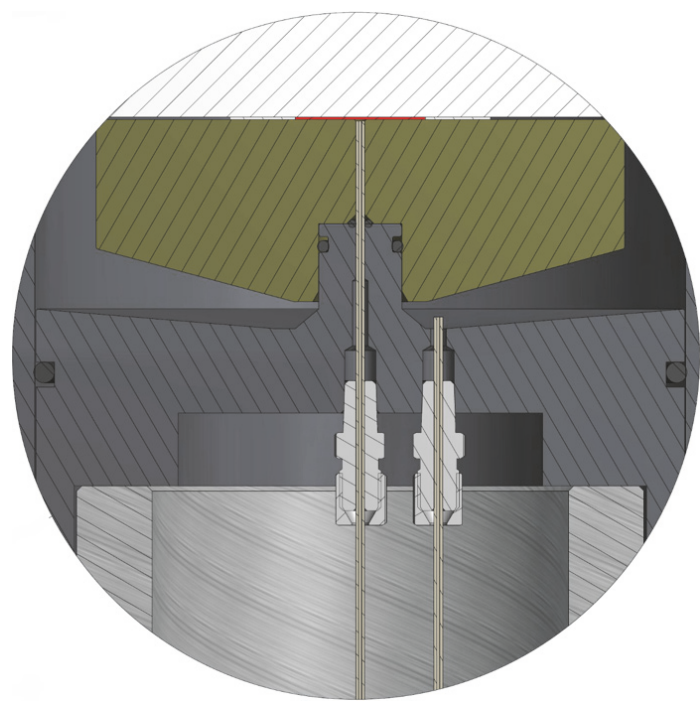

(c)

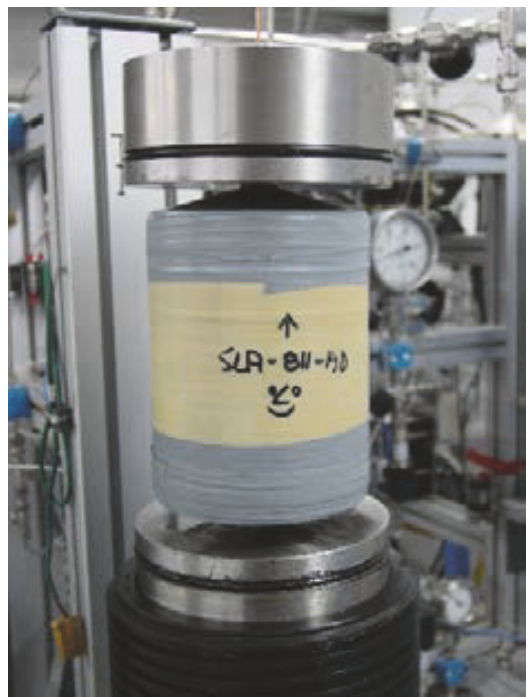

(e)

Figure 1: Advective displacement apparatus, (a) technical drawing, (b) schematic, (c) rock sample (white) with titanium coupling (green) and insert (dark grey) with details of O-ring seals and capillary for fluid injection (centre) and connection to confining fluid (off-centre), (d) insert with small O-ring sealing directly on the capillary for fluid injection, and (e) core sample mounted between inserts before lowering into pressure vessel. Inner diameter of this pressure vessel is $120 \mathrm{~mm}$; the diameter of the sample core is $101 \mathrm{~mm}$.

population on the clay exchanger, or mineral zonation in reactive systems.

(10) Redox-sensitive systems may be studied by imposing special precautions during sample preparation and sampling of the outflow and by introducing redoxsensitive tracers.

(11) Two-phase flow phenomena or the initial saturation state may also be tested (pore water uptake).

We tested in one or another way all of the potential applications mentioned above and are actively pursuing chemicophysical coupling phenomena, experimentally and by modelling approaches [11, 12]. Some select details are addressed in the technical description below.
2.3. Technical Description and Equipment. The principle design is that of a triaxial cell, but with a fixed length for the sample specimen (Figure 1). A rigid steel cylinder serves as pressure vessel, with a confining medium (water) that exerts a quasi-isostatic pressure on a cylindrical sample. Quasiisostatic refers to a small supported area that reduces the cross section on which confining pressure acts. Working with a confining pressure ensures a self-sealing system against the interior of a sample, a more reliable option compared to pressfits, glued-in samples, or other mechanical confinements.

The sample assembly (Figure 1(e)) comprises the cylindrical rock sample sandwiched between coupling pieces made of titanium. Porous filter discs are placed between the sample and the coupling pieces. The assembly is sealed against the 
confining medium by an inner layer of Teflon tape covered by a latex sleeve (details below). The assembly becomes selfsealing once the confining pressure is ramped up.

The sample assembly is mounted between two steel inserts (Figures 1(a) and 1(b)) and lowered into the pressure cylinder (Figure 1(e)). Conventional O-rings seal the enclosed confining medium against the pressure vessel and against the sample assembly (Figures 1(c) and 1(d)). A spindle (Figure 1(a)) adjusts to the length of a sample assembly, and normally no extra force is imposed initially on the sample by the spindle.

An artificial pore water is injected and collected with thin PEEK (polyether ether ketone) capillaries (1/16" OD, $1.6 \mathrm{~mm}$ ). These capillaries are fixed and fed through the sliding inserts by means of Swagelok fittings (Figure 1(c)), and an additional small O-ring is sealing the capillary at the contact point of the sample assembly and the inserts (Figure 1(d)) to avoid any stagnant dead volume of the percolating fluid.

Porous filter discs are a critical component that are required to distribute and collect the in-flow and outflow to and from a sample. A number of considerations determine the choice of filter material: minimizing pore volume is commonly desirable as well as chemical inertness. Porous Teflon is limited in stiffness and will collapse slowly when used at high confining pressures (above $2 \mathrm{MPa}$ ). A clever work-around is to use a thin porous Teflon filter with 3-5 small titanium frits as inserts that support the load. In this way, a filter thickness of $0.5 \mathrm{~mm}$ or less may be achieved with a diameter up to $120 \mathrm{~mm}$. Alternatives may be woven filters made from very thin titanium wire, or a grooved adapter surface in case of stiff rock like granite. Thick filters $(>1 \mathrm{~mm})$ are commonly not practical in applications for low-permeability rocks due to the significant dead volume compared to transport rates.

Steel cylinders for the pressure vessel are easiest manufactured from ready-made seamless precision steel tubes, honed, and centred on the inner diameter, such as used for hydraulic applications. Only an outer thread has to be machined such that caps can be screwed on at top and base (Figure 1(a)). Maximum pressure is limited by the maximum hoop stress that can be applied to the cylinder. A safe design is to hold maximum pressure of bottled argon or helium, for example, $20 \mathrm{MPa}$, but 50-100 MPa may also be reached with conventional O-ring sealing techniques. All our plumbing not contacted by pore fluids (for hydraulics) are made from stainless steel Swagelok components, using $6 \mathrm{~mm}$ and $1 / 8^{\prime \prime}$ $(3.2 \mathrm{~mm})$ product lines, and needle valves. These components are rated $>20 \mathrm{MPa}$ pressure.

Chemically inert materials are used for all fluid-wetted parts, including capillary tubing, valves, and fluid containers. Components from analytical equipment are readily available and include PEEK tubing, PEEK valves (used in high pressure liquid chromatography, e.g., Vici/Valco, Rheodyne, IDEX), and internally coated stainless steel tanks (sold as sampling cylinders, e.g., from Swagelok).

Sampling of aliquots from the outflow is conveniently done by small syringes directly connected to the outflow capillary; this protects samples from atmosphere. Syringe friction is significant (up to $0.1 \mathrm{MPa}$ for a $1 \mathrm{ml}$ standard O-ring sealed plastic syringe) when working at small hydraulic gradients. Alternatively, a gas-pressured back-pressure system can be implemented.

Controls include generation of the hydraulic confining pressure by bottled argon in a gas/liquid tank partially filled with the confining medium. This simple system also provides an effective buffering against pressure changes induced by temperature changes. The infiltrating artificial pore water is easiest driven at constant-pressure condition imposed by bottled He in a polymer-coated steel cylinder, with a capillary connecting to the infiltration apparatus via an injection valve. Using a 6-port two-position valve allows switching between two different fluids (e.g., one traced), or refilling the fluid reservoir at pressure, with a HPLC pump, for example. Helium is a good choice because of its small solubility and small pressure-dependence of solubility. Using argon or nitrogen leads to degassing in the experimental core that is subjected to a substantial hydraulic gradient.

We monitor all pressures and temperatures via data acquisition but do not use computer-control for long-term experiments, computers being far less reliable compared to hydraulic systems.

The pressure vessel may conveniently be heated or cooled by an outer heater jacket or cooling coil. A copper coil (or any other tubing material) in combination with a circulation heater/cooler is an easy way to thermostat or moderately heat or cool an experiment (indicated in Figure 1(a)).

Main operational safety issues relate to compressed gases, compressed liquids, and electric components. Most of our equipment is designed to hold full bottled gas pressure $(20 \mathrm{MPa})$, except for fluid/gas reservoirs that are generally rated to somewhat lower pressures (12-34 MPa for Swagelok sampling cylinders). Safety heads or pressure relieve valves protect such containers according to regulatory requirements.

A number of parameters for the outflowing fluid are conveniently measured in-line, including $\mathrm{pH}$, electric conductivity (EC), and redox potential (Eh). Except for conductivity, electrode measurements cannot be performed continuously over extended time, but small flow-through cells can be connected to a stream switching valve and activated for select periods of time, bracketed by calibration before and after in case of $\mathrm{pH}$. Convenient cells for EC are those used in ion-chromatography instruments, for example, as the one used by Metrohm (Figure 2(a)) that can be connected directly to $1 / 16^{\prime \prime}$ PEEK capillaries without dead volume and in conjunction with a Metrohm conductivity meter connected to data acquisition. These small two-electrode EC cells are designed for relatively low conductivities (large cell constant of ca. 10) and the conductivity meter has to be capable of using combinations of conductivity range and cell constants beyond what is common practice. Small flowthrough cells for $\mathrm{pH}$ are scarce on the market, and we fabricate ours in-house from POM (polyoxymethylene) and semi-micro-combination electrodes with tip diameters of 2$3 \mathrm{~mm}$, immersion depth of $4-6 \mathrm{~mm}$, and a flushed volume of 20-30 $\mu \mathrm{l}$ (Figure 2(b)). It is critical to provide a relatively soft seal above the electrode tip, and the mounting is very fragile. 


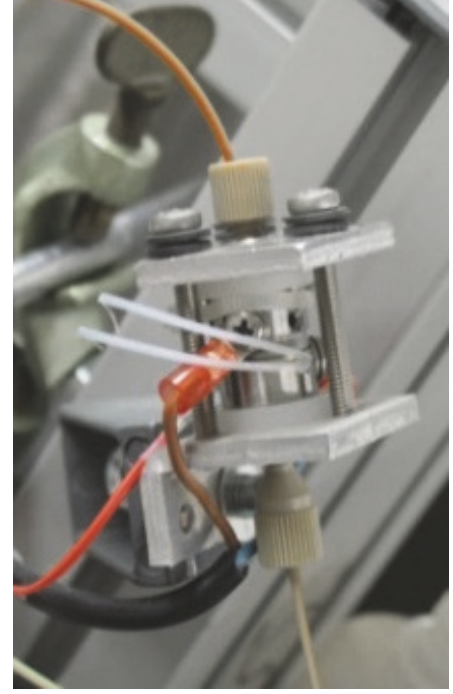

(a)

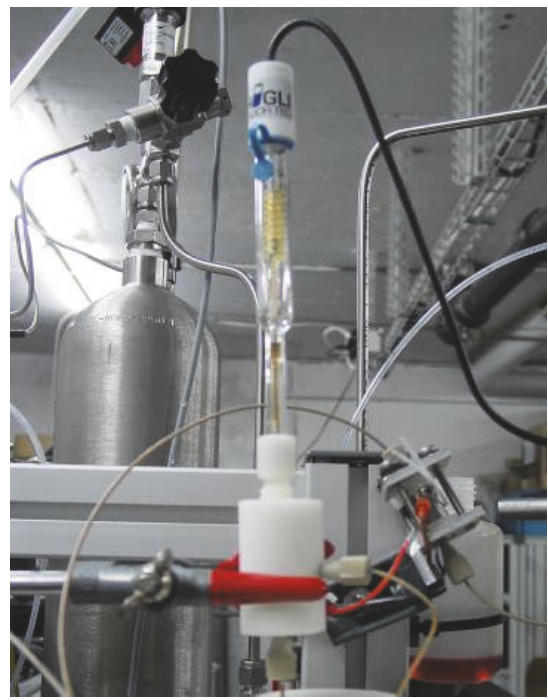

(b)

Figure 2: Flow-through cells for (a) electric conductivity and (b) $\mathrm{pH}$.

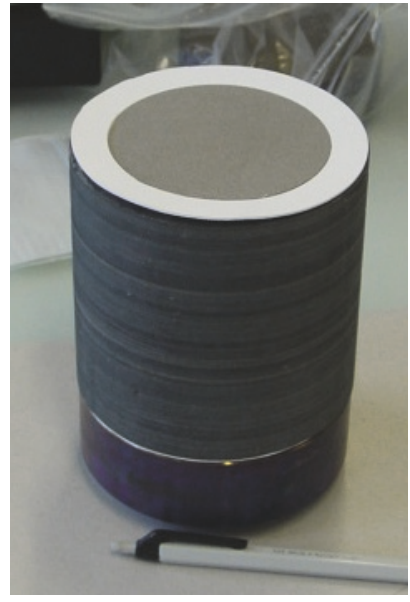

(a)

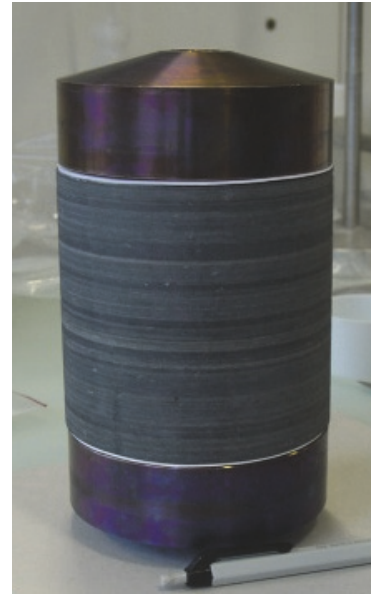

(b)

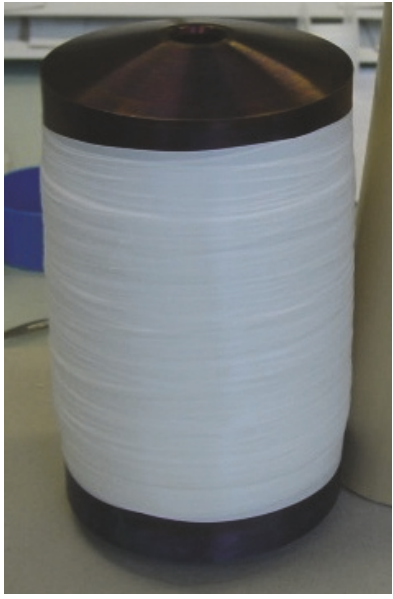

(c)

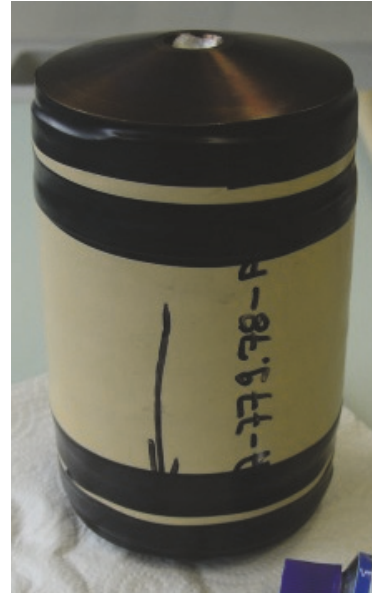

(d)

FIGURE 3: Stages of sample preparation: (a) rock core with porous filters at top and base, (b) placed between titanium couplings, (c) wrapped with Teflon tape, and (d) covered with latex sleeve. See text for details.

Using a 6-port stream switching valve, the ports for flushing and calibration can be set up in combination with the fluid outflow from the experiment.

Tests with measuring Eh in-line have been a mixed success. More often than not, only partly reducing conditions are obtained even over several weeks of flowthrough time, but never as low as might be expected from the sulphate/sulphide/pyrite/Fe-carbonate (or silicate) buffer imposed by most argillaceous rocks. This is likely due to the experimental set-up being not sufficiently gas-tight without converting to a more sophisticated construction. We made some tests using a very small-volume $(10-20 \mu \mathrm{l})$ cell from Metrohm used normally as a three-electrode amperometric detector, just using a $\mathrm{Ag} / \mathrm{AgCl}$ flat-base reference electrode, and either an $\mathrm{Au}$ or Pt measuring electrode.
2.4. Sample Preparation and Setting Up an Experiment. A requirement is a sample with preserved moisture content and that has been exposed to atmosphere for as little as possible. A cylindrical segment is dry-cut (or wet if permissible) with planar surfaces that are parallel and perpendicular to the cylinder axis. Total sample mass and dimensions are measured. Off-cuts are kept for determining chemicophysical parameters as a precharacterisation. We modified a mitre saw normally used for wood by fitting a diamond blade used normally for dry cutting. This is extremely efficient, precise, and inexpensive and cuts all materials but requires an industrial vacuum device for dust collection.

The sample core is placed between titanium coupling pieces (Figure 3), with filters in between. In the example shown (Figure 3(a)), a $1 \mathrm{~mm}$ thick porous titanium filter is 
used ( $85 \mathrm{~mm} \mathrm{DM}$ ) with a Teflon ring to match the sample diameter of $101 \mathrm{~mm}$. The assembly is sealed against confining pressure by an inner layer of Teflon tape (Figure 3(c)) covered by a latex sleeve (Figure 3(d)), sealed against the coupling parts with silicone sealant, and taped to ensure a tight initial seal.

Starting an advective displacement experiment includes loading the sample assembly into the pressure vessel (Figure 1(e)), filling in the confining fluid (water), and ramping up confining pressure, typically $1-2 \mathrm{MPa}$ above the desired injection pressure. Tightness of the sealing system of the rock sample (Figure 3) can be affirmed by leaving the system pressurized overnight, but without in-flow and outflow capillaries connected. We use the injection valve to evacuate the dead space in the filter disc and capillaries, switching directly from vacuum to the pressurized artificial pore water to start fluid injection. Likewise, the dead space in the fluid outflow part can be evacuated or flushed with argon with the help of a switching valve, before connecting to the sampling syringe. It may take several days until the head space is flushed with the displaced pore water, and arrival of outflow is first indicated by a jump in the electrical conductivity measured in-line (Figure 2(a)). Typical flow rates range from 0.05 to $0.5 \mathrm{ml} /$ day for argillaceous rock samples of $8-12 \mathrm{~cm}$ length and hydraulic heads of $2-8 \mathrm{MPa}$, corresponding to head gradients of $1.7 \cdot 10^{3}-10 \cdot 10^{3} \mathrm{~m} / \mathrm{m}$.

2.5. Tracers Used in Advective Displacement Experiments. A good frame of reference for transport is the use of a water tracer (deuterium, tritium) in all experiments. An anionic tracer should also be used, because anions get transported faster compared to water in clayey rocks due to a distinct anion exclusion effect that reduces the cross-sectional area available for transport and, hence, increases the average linear velocity. Choices are limited if the in situ anionic minor components are of interest $\left(\mathrm{Br}^{-}, \mathrm{I}^{-}\right.$, and low-molecular-weight organic acids). The anions contained in the undisturbed pore water can be observed as breakout tracers that elute from in situ concentration towards zero if the artificial pore water is tracer-free. Of concern are potential microbially induced redox reactions that may affect concentrations of anionic complexes such as sulphate and nitrate, particularly in the presence of dissolved natural organic matter. Many more choices of radionuclide tracers are available when working in a hot-laboratory environment.

Optical tracers, such as fluorescent dyes, are of little use in clay-rich rocks because these large molecules become retarded by sorption and/or potentially by ion filtration. It is safest to use tracers that resemble common pore water components, because testing for the suitability of a tracer at low transport rates is extremely time consuming.

2.6. Analytical Techniques. Sampling strategy for pore water displacement aims at collecting 3-5 small samples that are largely unaffected by the injected artificial pore water, such that ideally a concentration plateau can be confirmed and the onset of the anion tracer breakthrough detected. Sampling for obtaining breakthrough behaviour of tracers requires frequent early samples for inert tracers (water, anions) and far less frequent samples for late times or reactive components. A combination of in-line measurements and laboratory measurements of the sampled aliquots needs to be tailored to each experiment.

The parameters of interest involve those that can be measured in-line by electrodes with very small flow-through cells (electric conductivity, $\mathrm{pH}$, and redox potential, details above). Those measured on small aliquots in automated analysers include ion chromatography (major anions, cations, and some carboxylic acids), ICP-OES ( $\mathrm{Si}, \mathrm{Al}$, and some minor components such as Sr), carbon IR analyser (TIC, TOC, and NPOC), micro titration (total alkalinity), and cavity ringdown spectroscopy (CRDS, water stable isotopes, and some others). Analytical techniques need to be optimized for small sample volumes of $0.5-2 \mathrm{ml}$, but still allowing for the measurement of most of the desired parameters listed above. Key are an optimized dilution strategy, an appropriate workflow, and optimized autosamplers handling small-volume vials, apart from trained analytical personnel. More details on our analytical instruments and how this is implemented are given in some of our publicly accessible technical reports $[13,14]$.

2.7. Beyond Conventional Core Infiltration. We recently built core infiltration equipment from polymer plastics such that running experiments can be recorded periodically by Xray computed tomography, and so progress of fluid-rock interaction can be monitored, for example. We first used this in combination with medical X-ray CT and samples of compacted bentonite reacting with cementitious fluids $[15,16]$. The pressure vessel was fabricated from carbon fibre fabric, with fibre directions oriented to hold hoop stress and longitudinal stress. Threaded sleeves were made from fabric-reinforced epoxy polymer and glued onto the carbon fibre cylinder. Other components were machined from POM (polyoxymethylene). The maximum pressure is $5-10 \mathrm{MPa}$, limited by O-ring seals that are difficult to implement due to lack of stiffness and some creep.

A miniature version of a core infiltration cell was produced as a prototype for synchrotron X-ray CT experiments under geologic conditions, for example, fit for elevated pressure and temperature. The original cell was fabricated from a thick-walled optical glass cylinder (and later an aluminium cylinder) and caps made of reinforced PEEK [17] or stainless steel. The cell was designed for $20 \mathrm{MPa}$ confining pressure and an operation temperature up to $200^{\circ} \mathrm{C}$.

\section{Results and Discussion}

3.1. Anion Exclusion Effect and Controls on Pore Water Composition in Opalinus Clay. Anion exclusion effects are well known from soils, compacted bentonite, and argillaceous rocks [18] and are pronounced even in rocks that are not overly rich in clays, such as argillaceous limestone. The effect is largely electrostatic caused by clay surfaces (inner, outer) with permanent negative charge, such as in smectites, illite, and illite/smectite mixed layers. The effect is stronger at low ionic strength and vanishes at very high salinities as would be expected from Poisson-Boltzmann theory. One corollary is that salinities calculated form aqueous leaching and water 


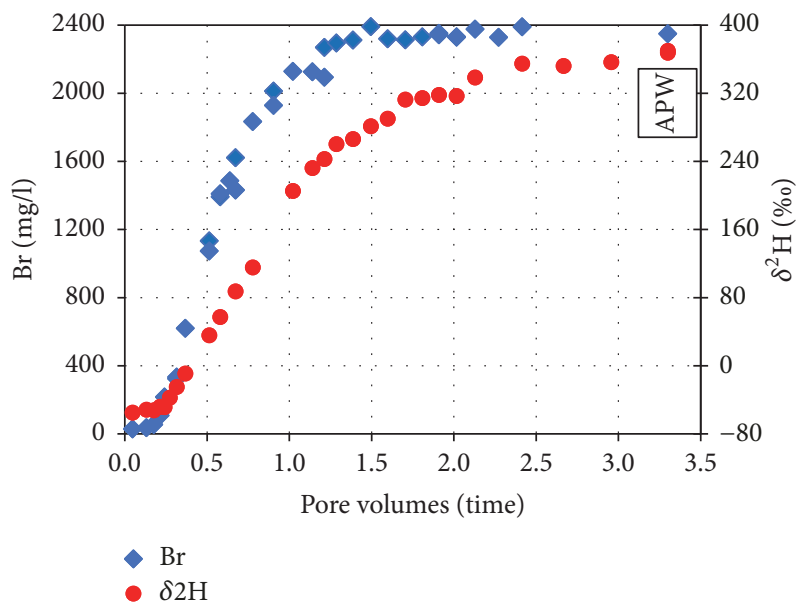

(a)

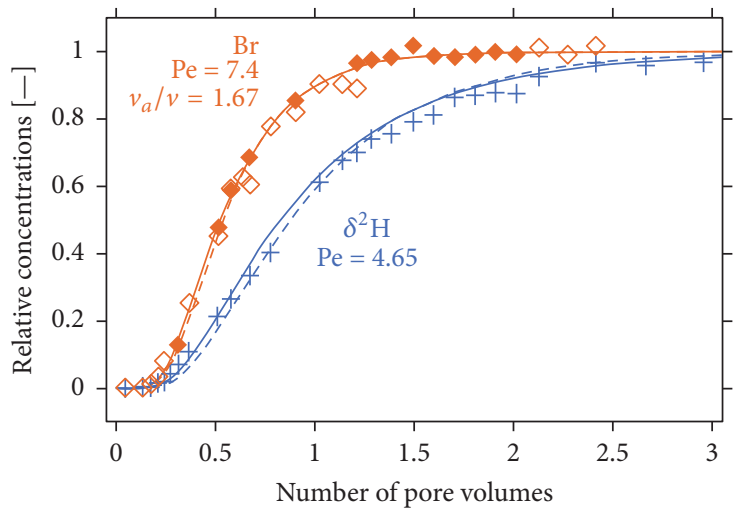

(b)

Figure 4: (a) Breakthrough of deuterated water and bromide versus time expressed as pore volume (water content); (b) modelled breakthrough curves with optimum values for Péclet numbers and the ratio of the anion average linear velocity relative to water, $v_{a} / v$. The dashed lines are for a model including small mixing cells at both ends of the sample, representing the filter discs.

content measurements may underestimate true salinities by a factor of two or more, and this is relevant for the evaluation of speciation, mineral saturation, and sorption.

Mäder et al. [10] and Mäder and Waber [19] presented one of the first advective displacement experiments with a sample from Opalinus Clay (proposed host rock for radioactive waste in Switzerland) including some preliminary data of early aliquots and showed a partial breakthrough of deuterated water and bromide that indicated an earlier breakthrough for bromide relative to water (as $\delta^{2} \mathrm{H}$, Figure 4 ). This experiment started in March 2002 and is still ongoing. Complete data for the first 2.5 years represent the percolation of 3 pore volumes (based on total water content) and during this time a full breakthrough of deuterated water and bromide was observed. The artificial pore water contained $9.3 \mathrm{~g} / \mathrm{l}$ chloride, $1.4 \mathrm{~g} / \mathrm{l}$ of sulphate, and $2.3 \mathrm{~g} / \mathrm{l}$ of bromide as tracer. This was somewhat more saline than the earliest sample aliquots of the outflow $\left(9.0 \mathrm{~g} / \mathrm{l} \mathrm{Cl}, 1.4 \mathrm{~g} / \mathrm{l} \mathrm{SO}_{4}\right)$, but the associated ionic strength effects caused by such a gradient on anion exclusion are minor. The earliest sample aliquots contained $30-37 \mathrm{mg} / \mathrm{l}$ of bromide, representing the pore water [10]. Modelling of the bromide and water tracer breakthrough with the one-dimensional advection-dispersion equation (Figure 4(b)) resulted in a ratio of linear average velocity for bromide relative to deuterated water of 1.67 and experimentscale Péclet numbers of $7.4(\mathrm{Br})$ and $4.65\left(\delta^{2} \mathrm{H}\right)$. The anion exclusion effect derived from modelling and expressed as chloride-accessible porosity fraction is 0.6 assuming this simple two-porosity model. The hydraulic conductivity of this sample (perpendicular to bedding) is $2.1 \cdot 10^{-13} \mathrm{~m} / \mathrm{s}$. Full details of the modelling are given in an unpublished technical report (Mäder et al. 2008).

Equilibrium speciation modelling of the early extracted aliquots (data tables in [10]) reveals a slight oversaturation with respect to calcite $(\mathrm{SI}=0.2)$ that can be corrected for a presumed $\mathrm{CO}_{2}$-outgassing effect, and this yields a partial pressure of $\mathrm{CO}_{2}$ of $10^{-2.6}-10^{-2.9}$ bar. Laboratory $\mathrm{pH}$ of the early aliquots of 7.4-7.5 are in agreement with one measured in-line of 7.5, just after taking a first sample aliquot. Of note are substantial initial concentrations of organic carbon $(60 \mathrm{mg} / \mathrm{l})$, and this was also observed in subsequent advective displacement experiments $[13,14]$ as well as in pore water squeezing [20]. Improved analytical capabilities revealed in later experiments that approximately half of the dissolved TOC is present in form of carboxylic acids like acetate, formate, and lactate. These concentrations are decreasing gradually with time below those seen in aqueous extracts back-calculated to pore water concentration but remain higher than measured from some boreholes used for longterm sampling of pore water [21]. The exact nature and mechanism of the production and release of carboxylic acids in these rocks and the relationship to the solid organic matter are not yet well understood.

Another distinct feature of this first experiment and all subsequent experiments with Opalinus Clay and adjacent clay-rich units is that the outflow appears to be buffered by celestite (Sr-sulphate) with calculated saturation indices within \pm 0.05 for most analyses, while sulphate is evolving gradually from the concentrations seen in the earliest extracts towards that in the artificial pore water. Partial data for Opalinus Clay is given by Mäder et al. [10], and sulphate elution from a different rock type is illustrated in Figure 5(b). The ubiquitous presence of celestite could not be demonstrated in these rocks and it is therefore possible that buffering by celestite may not operate due to its initial presence but may be controlled by precipitation at least near the outflow region of the Opalinus Clay sample. Mechanisms are not yet entirely clear, but the relevant ion activity product $\left[\mathrm{Sr}^{2+}\right] \cdot\left[\mathrm{SO}_{4}{ }^{2-}\right]$ may increase due to either a source for sulphate (small amount of pyrite oxidation) or a source for strontium, being displaced from the clay exchanger, for example. While the intrinsic coupling of carbonate/sulphate/ion-exchange (linking $\mathrm{pH}$, 


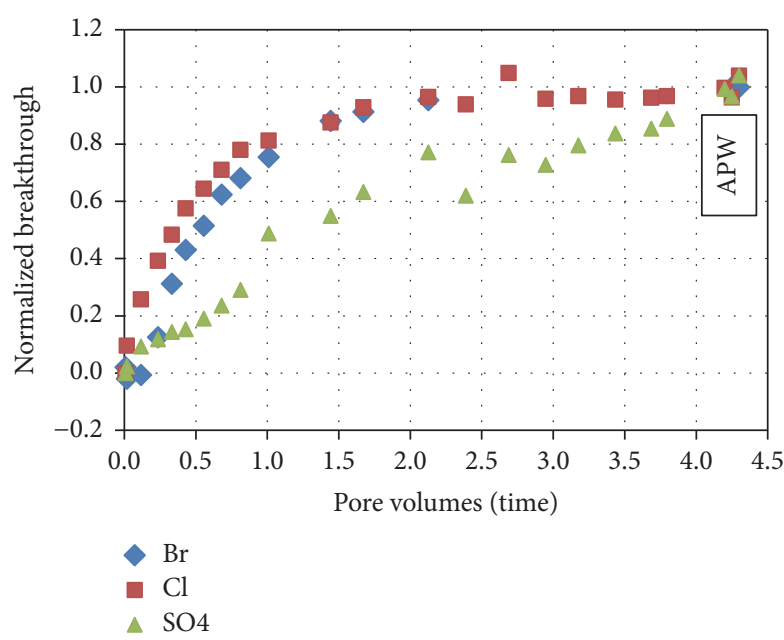

(a)

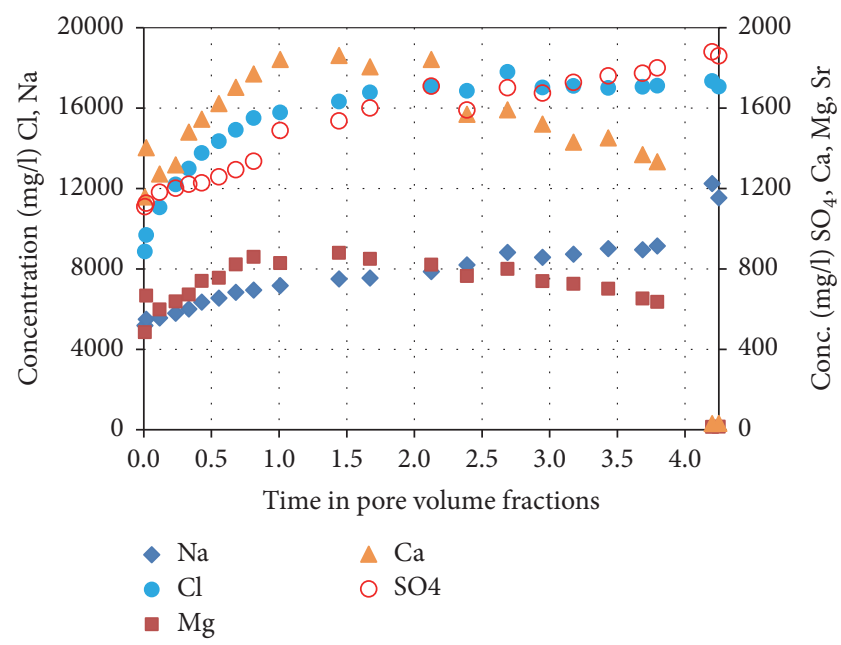

(b)

FIGURE 5: (a) Breakthrough of bromide, chloride, and sulphate in an argillaceous limestone, with experimental time converted to pore volumes (water content); (b) elution of select cations for the same experiment. The composition of the injected artificial pore water (APW) is indicated on the right side.

alkalinity, $\left.\mathrm{P}_{\mathrm{CO} 2}\right)$ had been intensely discussed $[3,5,6]$, the role of strontium in this context had been given far less attention.

3.2. Ion-Specific Transport Properties in an Argillaceous Limestone: Small Difference between $\mathrm{Br}^{-}$and $\mathrm{Cl}^{-}$. There is some debate about possible small differences between the transport properties (diffusion coefficient) of bromide and chloride. A measured bromide/chloride ratio near that of seawater is used as a strong indication of a marine origin of such a water. In the context of characterising pore water composition, tracer profiles, and water/rock interaction in a clay-rich Mesozoic sequence ([13], Effingen Member at Gösgen, Switzerland), one of the advective displacement experiments started with a mismatch in salinity between the injected artificial pore water and the in situ pore water as seen in the earliest aliquots. The injected pore water contained almost twice as much chloride as the earliest extracts $(17 \mathrm{~g} / \mathrm{l}$ versus $9 \mathrm{~g} / \mathrm{l})$ with sulphate values of $1.9 \mathrm{~g} / \mathrm{l}$ versus $1.1 \mathrm{~g} / \mathrm{l}$. The clay content of this argillaceous limestone is $20 \%$ (6\% illite/smectite mixed layers), the water-content porosity $5.6 \%$, and the hydraulic conductivity $1.3 \cdot 10^{-13} \mathrm{~m} / \mathrm{s}$. The anion-accessible porosity fraction is 0.64 , obtained by comparing the concentration of the early extracted aliquots with those of aqueous leachates back-calculated to the water content. The experiment was continued for 420 days. Full details are given in Mazurek et al. [13].

While the relatively strong salinity gradient due to the injection of a much more saline water has an effect on the anion exclusion extent, both bromide and chloride should be affected to the same extent. The breakthrough data for $\mathrm{Br}^{-}$and $\mathrm{Cl}^{-}$(Figure 5(a)) show a small but distinct and wellresolved difference between the two anions, with chloride being eluted a bit earlier than bromide. Such a behaviour demonstrates that there is indeed a small difference between the effective diffusion coefficients of $\mathrm{Cl}^{-}$and $\mathrm{Br}^{-}$in such a rock. Bromide elutes initially at $35 \mathrm{mg} / \mathrm{l}$ and is gradually flushed out until below detection limit after two pore volumes of transport. The breakout data is inverted and presented as breakthrough in the figure. In contrast to conservative anions, sulphate clearly elutes as a reactive component (Figure 5(b)). This data has not yet been modelled, one objective being the quantification of the difference in the apparent diffusion coefficient of bromide and chloride. Given enough geologic time, even a small difference in transport properties should lead to deviations from a seawater ratio simply by outdiffusion against a freshwater boundary, for example.

Chloride obviously elutes before bromide (Figure 5) indicating that either the transport-accessible pore volumes are different, the ion size/mobilities are different, or some form of different chemical interactions exists. The electrophoretic mobility of chloride [22] and thus the selfdiffusion coefficient in water (e.g., [23]) is smaller than that for bromide by ca. $2 \%$. On the other hand, the ionic radius (crystallographic/effective) of chloride $(0.167 / 0.181 \mathrm{~nm})$ is distinctly smaller than that for bromide $(0.182 / 0.196 \mathrm{~nm})$, but the hydrated radii are quite similar [24], compared to bromide being somewhat larger (ca. 0.330 versus $0.320 \mathrm{~nm}$, but overlapping data range), with a smaller hydration number for bromide (ca. 6 versus 8). If ion size is key, then the larger bromide may be retarded relative to chloride due to a "tortuosity effect" (pore size and shape, pore throat, size). The same type of preferential transport of chloride was observed by $\mathrm{Al}$ et al. [25] examining different ways of extracting dissolved pore water components from Opalinus Clay (and other clay rocks). These authors also discuss the potential role of ion pair formation that may be different between the two species.

The elution of cations (Figure 5(b)) is still far from equilibrium after percolation of four pore volumes. It is evident that $\mathrm{Ca}$ and $\mathrm{Mg}$ are displaced from the clay exchanger, and $\mathrm{Na}$ is retained. It should be possible to model such an 


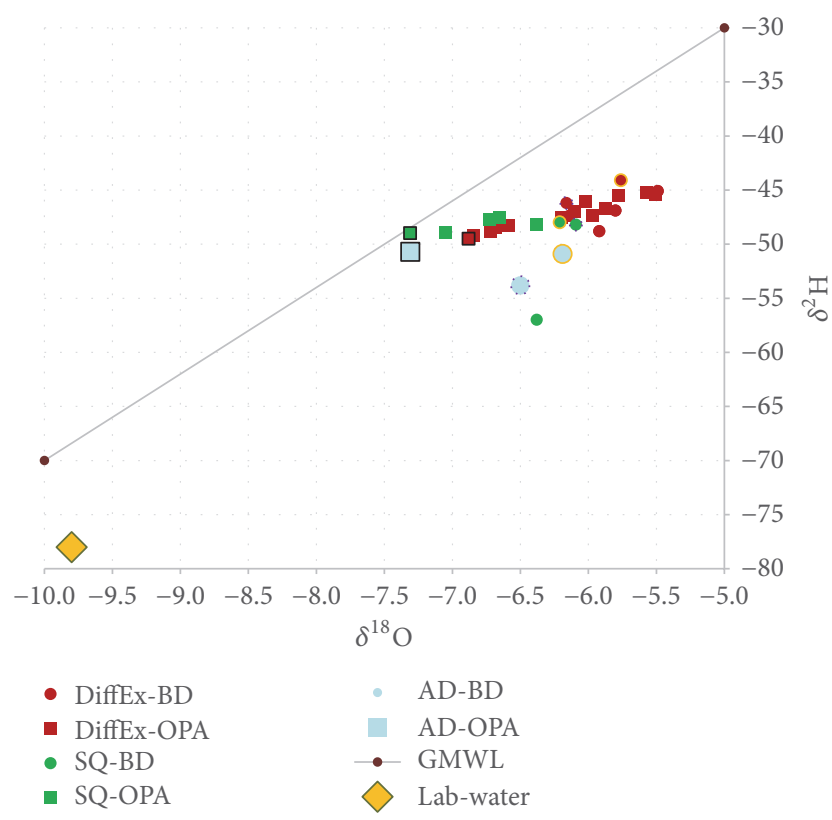

FIGURE 6: Water stable isotope composition of samples from advective displacement, diffusive exchange, and pore water squeezing from samples of Opalinus Clay (OPA) and Brown Dogger (BD). Samples from similar depth as advective displacement samples are correlated by different borders around symbols (black, orange, and dotted). Errors on $\delta^{18} \mathrm{O}$ are $0.15-0.2 \%$ and $1.5-2 \%$ for $\delta^{2} \mathrm{H}$, but precision is much better for samples measured in the same analytical sample series. DiffEx: diffusive exchange; SQ: squeezing; $\mathrm{AD}$ : advective displacement; GMWL: global meteoric water line.

experiment with a multicomponent reactive transport model and so gain more insight into the ion-exchange process and its coupling to the sulphate and carbonate system.

3.3. Water Stable Isotope Data Obtained from Advective Displacement Experiments. Advective displacement is not an efficient method for obtaining substantial amounts of water stable isotope data such as required to establish formationscale profiles. More efficient are diffusive exchange, distillation, or possibly squeezing. Profile data from diffusive exchange and squeezing and three samples from advective displacement are compared (Figure 6). The profile is from the Schlattingen geothermal well including a clay-rich Mesozoic sequence in Northern Switzerland. A full mineralogical and chemicophysical data set and methodology are included in Wersin et al. 2013 and 2016 [14, 26], except for the results from advective displacement experiments that are only partially included. Two units are examined in detail, Opalinus Clay and the overlaying Brown Dogger, both of Lower Jurassic age. Samples for diffusive exchange and squeezing that are located closest to the advective displacement samples are marked by coloured borders around symbols in Figure 6 . Diffusive exchange data define a linear trend inclined relative to the global meteoric water line. For Opalinus Clay, near-by samples subjected to squeezing and advective displacement are located on this trend but are shifted to more negative $\delta^{18} \mathrm{O}$ (by $0.5 \%$ ) relative to diffusive exchange, and also slightly more negative $\delta^{2} \mathrm{H}$ in case of advective displacement. For Brown Dogger, advective displacement data are also shifted to more negative values and so is one squeezing experiment but not the other one.

A likely reason for the isotope shift seen in the advective displacement experiment is that the sample cores were cut with a diamond saw using a small amount of tap water (composition indicated in Figure 6) that was blown off afterwards. At that time, the intention was not to derive reliable isotopic data, and our dry cutting facility was not yet implemented. It appears that by applying a more appropriate sample preparation water stable isotope data consistent with diffusive exchange may be obtained from advective displacement. The focus on the water stable isotopes in advective displacement is normally on using an artificial pore water with a large positive $\delta^{2} \mathrm{H}$ in order to detect and record breakthrough, and this is not very sensitive to the exact value in the initial samples.

\section{Conclusions}

Equipment developed for advective displacement and core infiltration experiments is relatively complex but based on simple hydraulic components, a robust design, and augmented by a careful choice of fluid-wetted materials. Key is a well-organized strategy for sampling, sample preservation, and sample preparation.

Advective displacement and its extension as a core infiltration experiment can yield a large amount of data to constrain the composition of the pore water preserved in a sample and transport properties derived from breakthrough of tracers. Elution of reactive components subject to ionexchange or buffering by mineral equilibria (such as shown to operate for strontium and sulphate by celestite) requires extended experimental times of $>1$ year for such tight clayey rocks. Analytical methods have to strike a compromise between sample size (time resolution) and the number of components to be analysed. In-line analysis by electrodes (EC, $\mathrm{pH}$, and $\mathrm{Eh}$ ) in very small-volume flow-through cells is possible, but meaningful $\mathrm{Eh}$ measurements likely require a more elaborate protection from gas-diffusion and sample conditioning. Experiments with multiple tracers allow direct comparison to resolve small differences in transport properties of conservative components, such as shown to exist for chloride and bromide.

A comparison of pore water squeezing and advective displacement is currently hampered by very few available data gathered from the exact same rock samples. Both methods do induce certain artefacts. One issue is the observed salinity gradient obtained from subsequent squeezing aliquots [20], a consequence of anion exclusion, that render the composition of a squeezed aliquot sensitive to the chosen squeezing conditions. Both methods appear to mobilize organic compounds and this is evident in substantial concentrations of low-molecular-weight organic acids (early aliquots in advective displacement) that are much higher than what can be recovered in aqueous extracts or during long-term borehole sampling. Advective displacement is a destructionfree method whereas squeezing may provoke strain-induced dissolution of minerals such as carbonates. The extent of 
anion exclusion (chloride) obtained by both methods so far from clay-rich rocks of interest in Switzerland is in reasonable agreement (ca. 0.4-0.6 for anion-accessible/total porosity).

Possible improvements to sample preparation include efficient removal of the outer cylindrical surface by turning it on a lathe and by accurate dry cutting with diamond cutting facilities. This is expected to yield accurate water isotope composition for the early extracts that represent most closely the preserved pore water. In comparison to throughdiffusion experiments, core infiltration also provides information on hydraulic properties, and feedback of changes in porosity/permeability to transport can be resolved precisely.

\section{Conflicts of Interest}

The author declares that there are no conflicts of interest.

\section{Acknowledgments}

All new equipment was built in-house at our machine shop. Adrian Liechti and Thomas Siegenthaler provided their expertise and a number of students helped to assemble "the beasts." Thomas Gimmi performed the tracer transport modelling calculations for the first Opalinus Clay experiment. Nagra (National Cooperative for the Disposal of Radioactive Waste) partially supported and funded this research. Nick Waber was a very constructive discussion partner about pore water chemistry.

\section{References}

[1] A. Gautschi, "Safety-relevant hydrogeological properties of the claystone barrier of a Swiss radioactive waste repository: An evaluation using multiple lines of evidence," Grundwasser, vol. 22, no. 3, pp. 221-233, 2017.

[2] M. Mazurek, P. Alt-Epping, A. Bath et al., "Natural tracer profiles across argillaceous formations," Applied Geochemistry, vol. 26, no. 7, pp. 1035-1064, 2011.

[3] F. J. Pearson, D. Arcos, A. Bath et al., Mont Terri Project - Geochemistry of Water in the Opalinus Clay Formation at the Mont Terri Rock Laboratory, Geology Series No. 5, Reports of the Federal Office of Water and Geology (FOWG), 2003.

[4] F. J. Pearson, C. Tournassat, and E. C. Gaucher, "Biogeochemical processes in a clay formation in situ experiment: Part E Equilibrium controls on chemistry of pore water from the Opalinus Clay, Mont Terri Underground Research Laboratory, Switzerland," Applied Geochemistry, vol. 26, no. 6, pp. 990-1008, 2011.

[5] C. Tournassat, A. Vinsot, E. C. Gaucher, and S. Altmann, "Chemical conditions in Clay-Rocks," in Natural and Engineered Clay Barriers, Developments in Clay Science, C. Tournassat, C. I. Steefel, I. C. Bourg, and F. Bergaya, Eds., vol. 6, pp. 71-100, Elsevier, 2015.

[6] C. Tournassat, C. I. Steefel, I. C. Bourg, and F. Bergaya, Natural and Engineered Clay Barriers, Developments in Clay Science, vol. 6, Elsevier, 2015.

[7] L. R. Van Loon and J. Mibus, "A modified version of Archie's law to estimate effective diffusion coefficients of radionuclides in argillaceous rocks and its application in safety analysis studies," Applied Geochemistry, vol. 59, pp. 85-94, 2015.

[8] C. Wigger and L. R. Van Loon, "Importance of Interlayer Equivalent Pores for Anion Diffusion in Clay-Rich Sedimentary
Rocks," Environmental Science \& Technology, vol. 51, no. 4, pp. 1998-2006, 2017.

[9] P. Alt-Epping, C. Tournassat, P. Rasouli et al., "Benchmark reactive transport simulations of a column experiment in compacted bentonite with multispecies diffusion and explicit treatment of electrostatic effects," Computational Geosciences, vol. 19, no. 3, pp. 535-550, 2015.

[10] U. K. Mäder, H. N. Waber, and A. Gautschi, "A new method for porewater extraction from claystone and determination of transport properties with results for Opalinus Clay (Switzerland)," in Proceedings of the 11th International Symposium on Water-Rock Interaction, WRI-11, R. B. Wanty and R. R. Seal II, Eds., pp. 445-448, 2004.

[11] A. Yustres, A. Jenni, L. Asensio et al., "Comparison of the hydrogeochemical and mechanical behaviours of compacted bentonite using different conceptual approaches," Applied Clay Science, vol. 141, pp. 280-291, 2017.

[12] A. Jenni, T. Gimmi, P. Alt-Epping, U. Mäder, and V. Cloet, "Interaction of ordinary Portland cement and Opalinus Clay: Dual porosity modelling compared to experimental data," Physics and Chemistry of the Earth, vol. 99, pp. 22-37, 2017.

[13] M. Mazurek, H. N. Waber, U. K. Mäder et al., "Geochemical Synthesis for the Effingen Member in Boreholes at Oftringen, Gösgen and Küttigen," Nagra Technical Report NTB 12-07 Nagra, Wettingen, Switzerland, 2012.

[14] P. Wersin, M. Mazurek, U. K. Mäder et al., "Rock and porewater characterisation on drillcores from the Schlattingen borehole," Nagra Arbeitsbericht NAB 12-54 Nagra, Wettingen, Switzerland, 2013.

[15] F. Dolder, U. Mäder, A. Jenni, and N. Schwendener, "Experimental characterization of cement-bentonite interaction using core infiltration techniques and 4D computed tomography," Physics and Chemistry of the Earth, Parts A/B/C, vol. 70-71, pp. 104-113, 2014.

[16] F. Dolder, U. Mäder, A. Jenni, and B. Münch, "Alteration of MX80 bentonite backfill material by high-pH cementitious fluids under lithostatic conditions - an experimental approach using core infiltration techniques," Journal of the Geological Society, vol. 443, no. 1, pp. 281-305, 2017.

[17] F. Fusseis, H. Steeb, X. Xiao et al., "A low-cost X-ray-transparent experimental cell for synchrotron-based X-ray microtomography studies under geological reservoir conditions," Journal of Synchrotron Radiation, vol. 21, no. 1, pp. 251-253, 2014.

[18] S. T. Horseman, J. J. W. Higgo, J. Alexander, and J. F. Harrington, "Water, gas and solute movement through argillaceous media," Tech. Rep. 96/1. OECD/NEA, France, Paris, 1996.

[19] U. Mäder and H. Waber, "Characterization of Pore Water, Ion Transport and Water-rock Interaction in Claystone by Advective Displacement Experiments," Procedia Earth and Planetary Science, vol. 17, pp. 917-920, 2017.

[20] M. Mazurek, T. Oyama, P. Wersin, and P. Alt-Epping, "Porewater squeezing from indurated shales," Chemical Geology, vol. 400, pp. 106-121, 2015.

[21] A. Courdouan, I. Christl, S. Meylan, P. Wersin, and R. Kretzschmar, "Characterization of dissolved organic matter in anoxic rock extracts and in situ pore water of the Opalinus Clay," Applied Geochemistry, vol. 22, no. 12, pp. 2926-2939, 2007.

[22] J. Aupiais, "Electrophoretic mobilities of the isotopes of chloride and bromide ions in aqueous solution at $25 \circ \mathrm{C}$ and infinite dilution," Journal of Solution Chemistry, vol. 40, no. 9, pp. 16291644, 2011. 
[23] S. Koneshan, J. C. Rasaiah, R. M. Lynden-Bell, and S. H. Lee, "Solvent structure, dynamics, and ion mobility in aqueous solutions at $25 \circ$ C," The Journal of Physical Chemistry B, vol. 102, no. 21, pp. 4193-4204, 1998.

[24] P. R. Smirnov, "Structural parameters of the nearest surrounding of halide ions in the aqueous electrolyte solutions," Russian Journal of General Chemistry, vol. 83, no. 8, pp. 1469-1481, 2013.

[25] T. Al, M. Celejewski, and D. Barton, "Observations of variable geochemical porosity for $\mathrm{Cl}$ and $\mathrm{Br}$ from clay-rich sedimentary rocks," in Proceedings of the Book of Abstracts, 7th International Conference on Clays in Natural and Engineering Barriers for Radioactive Waste Confinement, pp. 106-107, Davos, Switzerland, 2017.

[26] P. Wersin, M. Mazurek, U. K. Mäder et al., "Constraining porewater chemistry in a $250 \mathrm{~m}$ thick argillaceous rock sequence," Chemical Geology, vol. 434, pp. 43-61, 2016. 

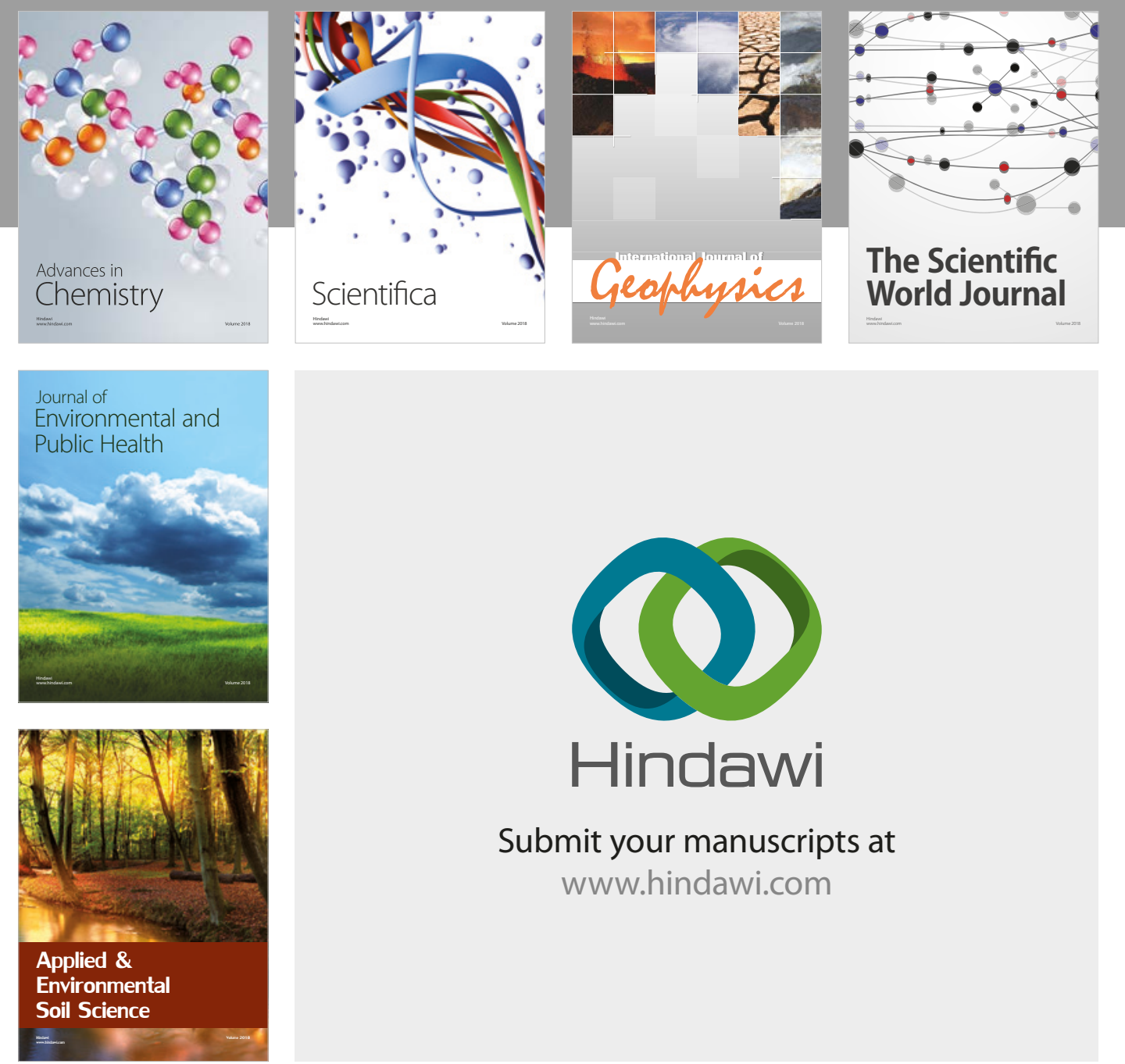

The Scientific

\section{World Journal}
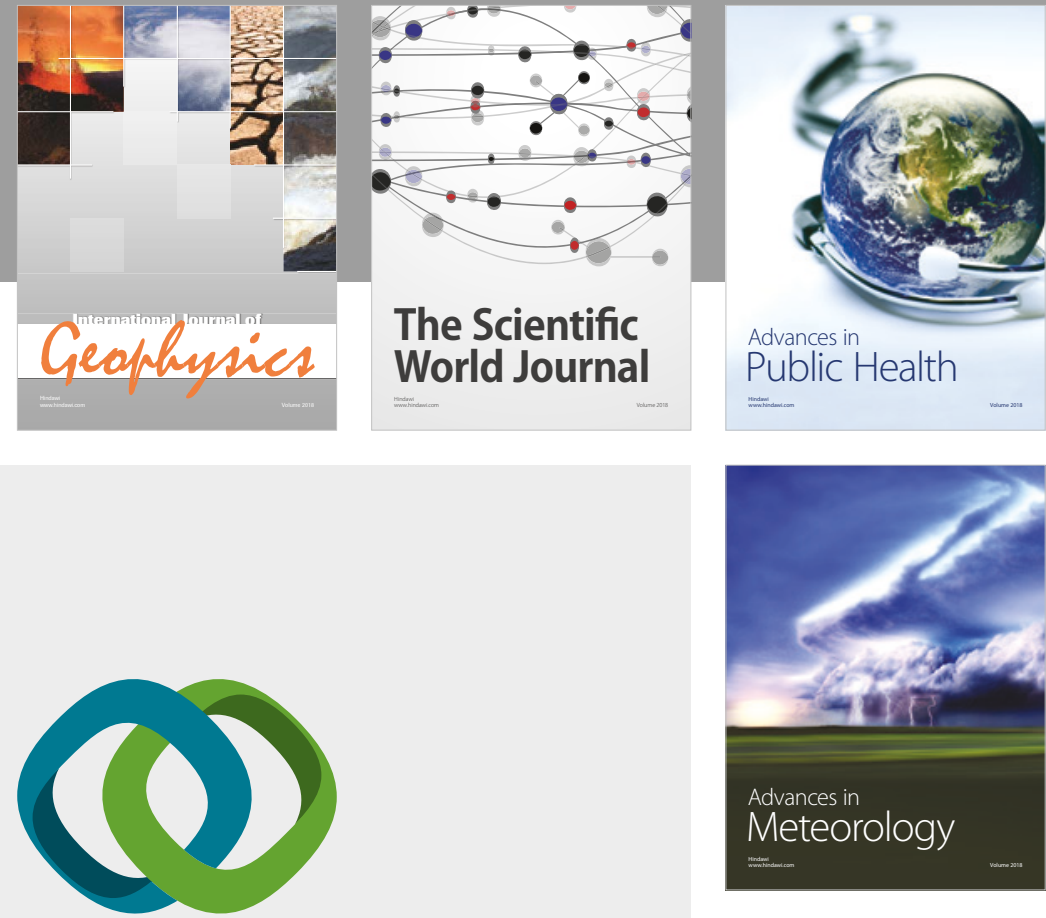

Advan

Public Health

\section{Hindawi}

Submit your manuscripts at

www.hindawi.com
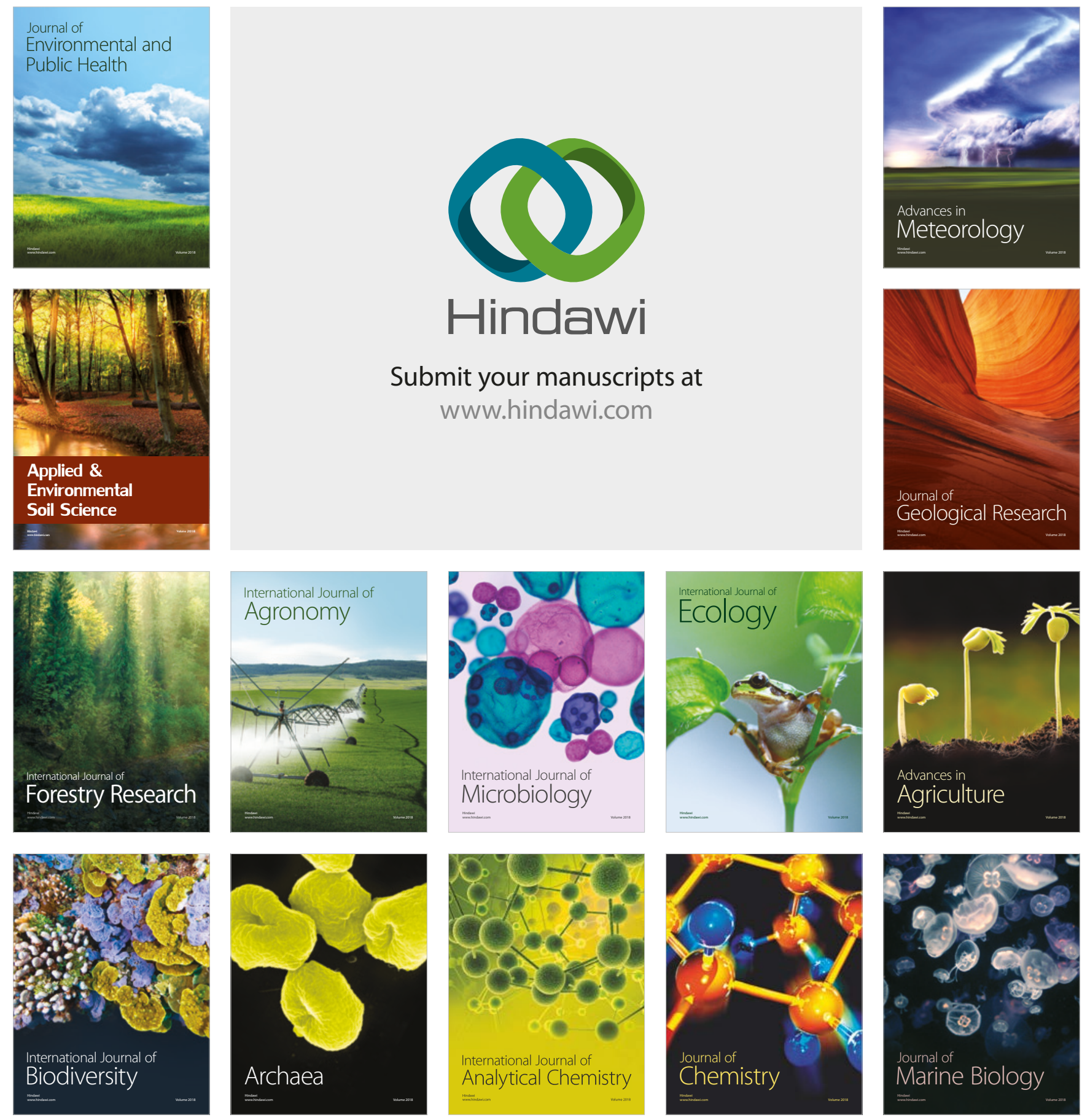